\title{
Detectors Capable Of High Countrates and High Speed Pulse Processing Electronics for X-Ray Spectroscopy
}

\author{
Joe Nicolosi, Michael Solazzi, Del Redfern
}

\section{EDAX Inc. Mahwah, NJ, USA}

Traditionally, $\mathrm{Si}(\mathrm{Li})$ detectors have been used in Energy Dispersive (X-ray) Spectroscopy (EDS). Indeed these have evolved to perform at very near theoretical limits of energy resolution. Typical specifications are 129 eV FWHM @ Mn Ka. Recently however, new technologies have opened new dimensions to EDS analysis. Commercial availability of the Silicon Drift Detector (SDD) has enabled collection of x-ray events at much higher countrates without significant resolution degradation. Whereas historically it has been difficult to produce high count rate conditions in micro-EDS (electron excited) analysis, with FEG columns and samples of high fluorescent yield it is now possible to achieve countrates in excess of what can be handled by $\mathrm{Si}(\mathrm{Li})$ detectors.

In the case of X-Ray (excited) Fluorescence (XRF) spectrometers high countrates have always been available to the detection system. The SDD is ideally suited as an EDXRF detector. With recent advances using poly-capillary optics to collect and focus incident beam flux onto micro-samples as small as $30 \mu \mathrm{m}$ one can also achieve high countrates in micro-XRF systems today.

In order to take full advantage of these high countrates, the counting electronics needs to operate at sub microsecond shaping times. In the latest designs of digital pulse processors (DPP II) it is not only possible to select short shaping times but use adaptive processing to make optimum use of time between pulses for filtering.

In this paper we will describe several applications, including fast x-ray mapping, where the use of high countrate capable SDD, in combination with high-speed digital signal processing used in x-ray microanalysis and x-ray spectroscopy. Data will also be presented where the SDD can provide better resolutions that $\mathrm{Si}(\mathrm{Li})$ detectors in the $>20,000$ count per second range.. 


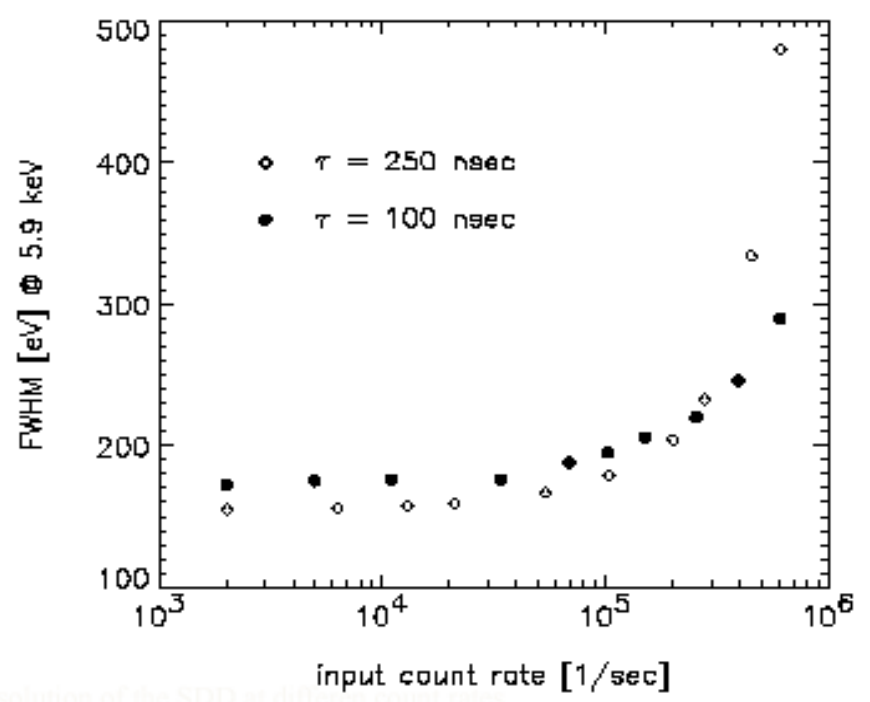

Energy Resolution of the SDD at different count rates

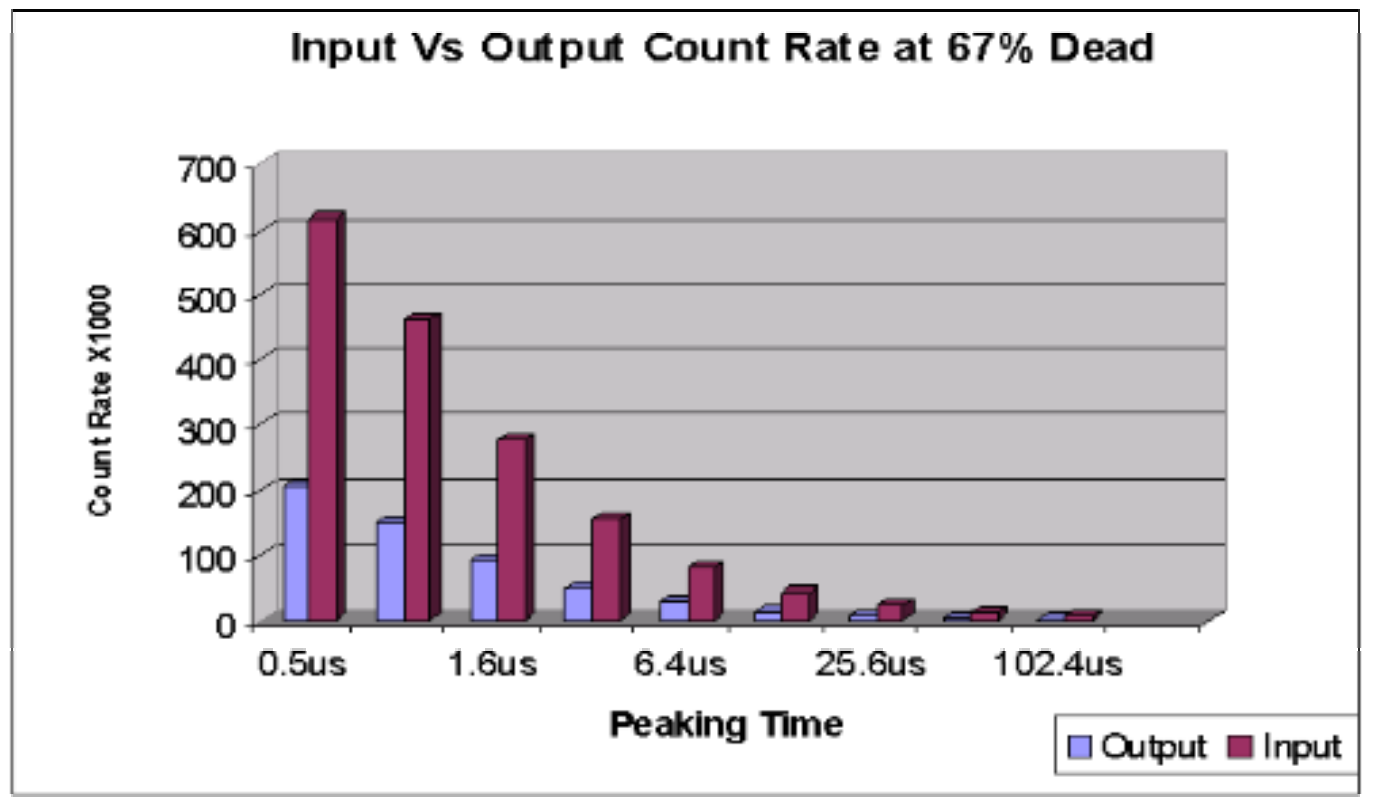

DPP II Input v Count Rate

\begin{tabular}{|l|l|l|l|l|l|l|l|l|l|}
\hline $\begin{array}{l}\text { Peak } \\
\text { Time }\end{array}$ & $\mathbf{0 . 5}$ & $\mathbf{0 . 8}$ & $\mathbf{1 . 6}$ & $\mathbf{3 . 2}$ & $\mathbf{6 . 4}$ & $\mathbf{1 2 . 8}$ & $\mathbf{2 5 . 6}$ & $\mathbf{5 1 . 2}$ & $\mathbf{1 0 2 . 4}$ \\
\hline Input & 616 & 462 & 277 & 154 & 82 & 42 & 21 & 11 & 5.4 \\
\hline Output & 203 & 152 & 61 & 51 & 27 & 14 & 7 & 3.5 & 1.8 \\
\hline
\end{tabular}

DPP II Input v Output Countrate 